\title{
KINEMATIC DEXTERITY ANALYSIS OF THE UPPER DUAL LIMB REHABILITATION ROBOT
}

\author{
Qing Sun ${ }^{1}$, Shuai Guo ${ }^{1}$, Leigang Zhang ${ }^{1}$, Sixian Fei ${ }^{1}$ \\ ${ }^{1}$ School of Mechatronic Engineering and Automation, Shanghai University, No.99 Shangda Road, \\ Baoshan District, Shanghai 200444, China \\ E-mails: qingsun93@163.com; guoshuai@shu.edu.cn; lgzhang@shu.edu.cn; fsx1993@shu.edu.cn
}

\begin{abstract}
The trajectory formulation of the rehabilitation robot is the key to rehabilitation training, and the kinematic dexterity of rehabilitation robot is the basis of the trajectory formulation. About the kinematic dexterity of rehabilitation robot, a mixing method of Monte Carlo and cell segmentation based on manipulability is proposed to obtain the spatial grid discrete diagram of kinematic dexterity of the rehabilitation robot, which provides a basis for the selection of rehabilitation robot training trajectory. Firstly, the workspace of the robot is obtained based on Monte Carlo method. Secondly, the cell segmentation method is used to segment the workspace. Then, the operating points in each grid are treated with the average of operability. Next, taking rehabilitation robot as the research object, the kinematic dexterity analysis is carried out by the mixture method of Monte Carlo and cell segmentation. Finally, dexterity simulation analysis is carried out by MATLAB, and the kinematic dexterity spatial grid discrete diagram of rehabilitation robot was obtained. At the same time, it also lays a foundation for making the trajectory formulation of the rehabilitation robot.
\end{abstract}

Keywords: Rehabilitation robot; Cell segmentation method; Manipulability; Dexterity.

\section{Introduction}

According to the World Health Organization (WHO), there are more than 10 million new stroke patients every year, among which 6 million patients will have lifelong disability [1]. According to the theory of motor relearning plan, the peripheral nerve injury caused by upper limb dysfunction can be restored by proper physical exercise and effective rehabilitation strategies. Researches have shown that the repetitive task training can effectively improve the rehabilitation effect of upper limb motor function [2]. Effective rehabilitation training trajectory requires good robot locomotion performance, which needs to get the kinematic dexterity distribution of robot in the workspace. Therefore, it is of great significance to study the kinematic dexterity of the robot for the trajectory formulation of the rehabilitation robot.

Many scholars have studied various aspects of robot dexterity and have proposed a number of dexterity indexes, including the manipulability [3], directional manipulability [4], the condition number [5] and the isotropic index [6], and so on. The conditional number and isotropic indexes are used as the index of ellipsoidal orientation consistency, and manipulability and directional manipulability are used as the index of robot locomotion. Chen et al. [7] adopted inverse kinematics to study the accessibility map of flexible assembled industrial dual limb robots, and designed the configuration of dual limb robots based on the accessibility map. Wagner et al. [8] presented an approach for the generation of reachability maps for cooperative processing with multiple robots, which was implemented for a setup with two industrial robot arms. In order to improve the accuracy of the generated reachability maps, the calculation was extended by a collision detection. In addition, the calculation time was slightly improved by the added collision detection. Tian et al. [9] proposed an improved method to solve the workspace dexterity of collaborative robots based on the concept of service ball. Each service ball needed to be traversed at each joint Angle to avoid the deficiency of the inverse kinematics method. Sun et al. [10] proposed a VMEIV (velocity manipulability ellipsoid intersection volume) index to analyse the kinematic dexterity of human-robot interaction. These researches on the robot kinematic dexterity make a great contribution. Researchers usually get the robot kinematic dexterity visualization through the traversal algorithm and the inverse kinematics segmenting workspace, while the solutions are relatively difficult to carry out the inverse kinematics solution and the traversal algorithm. There few researchers use the kinematic dexterity to study the robot trajectory formulation in the rehabilitation 
training. The main contribution of this paper is focus on the problem.

This paper studies the rehabilitation robot of the research group. Firstly, the DH (Denavit-Hartenber) coordinate system is established for the robot forward kinematics equation calculation. Secondly, the workspace of robot is obtained based on Monte Carlo method, and the workspace grid is discretized by cell segmentation method. Then, each operating point in the grid is solved by the Jacobian matrix to obtain the manipulability of each point, and the manipulability of each point is averaged. Finally, the manipulability of each grid in the workspace is normalized, and the kinematic dexterity distribution diagram of the rehabilitation robot is visualized by MATALB. In this study, a mixing method of Monte Carlo and cell segmentation based on the manipulability is adopted to obtain the spatial grid discrete diagram of the kinematic dexterity of the rehabilitation robot, which avoids the inverse kinematics solution and traversal algorithm of the robot and provides reference for the training trajectory formulation of the rehabilitation robot.

\section{The Discrete Grid of the Robot Workspace.}

\subsection{Workspace Solution based on Monte Carlo}

Nowadays, the Monte Carlo method [11] is used more and more common in solving robot workspace due to the rapid development of computer technology. The quality of Monte Carlo method depends on whether the generation of random functions is uniform. Therefore, this paper adopts the mixed congruence method to generate random numbers. The formula is as follow:

$$
\left\{\begin{array}{c}
X_{\mathrm{n}}=\bmod \left(A X_{\mathrm{n}-1}+C, M\right) \\
Y_{\mathrm{n}}=X_{\mathrm{n}} / M
\end{array}\right.
$$

where mod refers to the codependent function; $A$ refers to the multiplier; $C$ refers to the increment; $M$ refers to the norm; $X_{n}$ refers to the random variable that is uniformly distributed in the interval $(0, \mathrm{M}) ; Y_{n}$ refers to the random variable obeying uniform distribution in $(0,1)$, and has $n$ groups $(n<M)$ random numbers.

Obviously, in the above equation, the values of A, $\mathrm{C}$ and $\mathrm{M}$ parameters have $\mathrm{A}$ great influence on the selection of random functions. Reference [12] has proved that the random number sequence obtained when $A=1045, C=1$ and $M=230$ has a good statistical effect.
The joint angles $\theta_{i}$ of the robot are obtained by the mixed congruence method, which is known from Eq. (2)

$$
\theta_{\mathrm{i}}=\theta_{\mathrm{i}}^{\min }+\mathrm{Y}_{\mathrm{n}}\left(\theta_{\mathrm{i}}^{\max }-\theta_{\mathrm{i}}^{\min }\right)
$$

where $\theta_{i}$ refers to joint variable; $\theta_{\mathrm{i}}^{\max }$ refers to the upper limit of joint variable interval, and $\theta_{\mathrm{i}}^{\min }$ refers to the lower limit of joint variable interval; $\theta_{\mathrm{i}}^{\min } \leq \theta_{\mathrm{i}} \leq \theta_{\mathrm{i}}^{\max }, i=1,2 \ldots 7$.

The relationship between the end position of robot and the joints angle of robot can be obtained by the forward kinematics of the robot, which is known from Eq. (3)

$$
P=\left(p_{x}, p_{y}, p_{z}\right)=\left\{\begin{array}{l}
p_{x}=f_{x}\left(\theta_{1}, \theta_{2}, \cdots, \theta_{7}\right) \\
p_{y}=f_{y}\left(\theta_{1}, \theta_{2}, \cdots, \theta_{7}\right) \\
p_{z}=f_{z}\left(\theta_{1}, \theta_{2}, \cdots, \theta_{7}\right)
\end{array}\right.
$$

where $P$ refers to the position vector of the robot end-effector; $p_{x}, p_{y}, p_{z}$ represents the position coordinates component of the robot end-effector.

\subsection{The Grid Discrete of the Workspace}

The traversal algorithm is usually used to search and classify the database for the grid discretization in the workspace, and the calculation amount $\left(n_{x} \cdot n_{y}\right.$. $n_{z} \cdot S$ ) is obviously the number of grids divided multiplied by the number of samples in the database. Where $\left(n_{x} \cdot n_{y} \cdot n_{z}\right)$ is the segmentation numbers in the direction of $x, y, z$ coordinate axes, respectively, and $S$ is the sample numbers. As shown in Fig.1

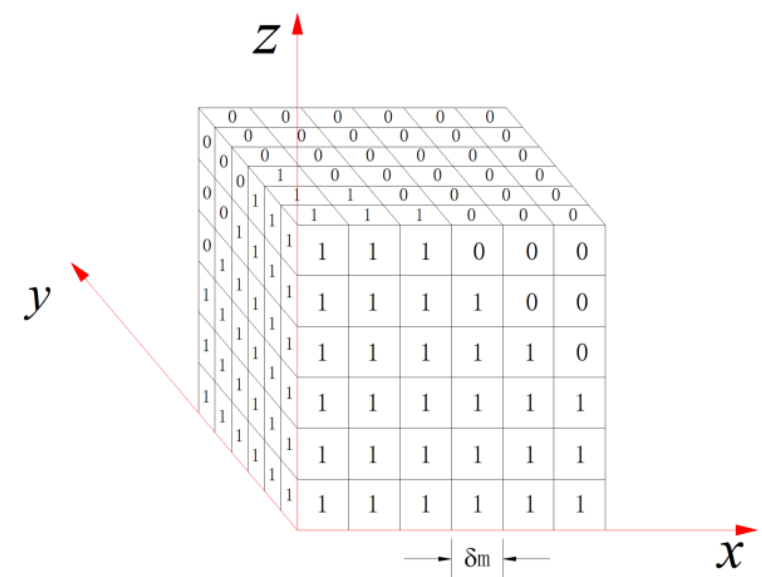

Fig. 1. Schematic diagram of grid dispersion

In order to reduce the computational burden of working space grid discretization, cell segmentation method is adopted in this study. The specific steps are as follows: 
Step 1: Divide the workspace into $n_{x}, n_{y}$, $n_{z}$ grids along $x, y, z$ coordinate axis, respectively;

Step 2: Reduce the three-dimensional coordinate to the one-dimensional coordinate. Take the $x$ coordinate of $S$ as the object, arrange along the grid in the direction of Axis $x$, and place the sample $S$ in the one-dimensional grid in the direction of axis $x$, so the calculation amount is $n_{x} \cdot S$.

Step 3: Arrange the sample $S$ of the grid on axis $x$ of one-dimensional coordinates along axis $y$. Then, sample $S$ is placed in two-dimensional grid on the plane $x y$, so the calculation amount is $n_{y} \cdot S$

Step 4: Arrange the sample $S$ of two-dimensional grid in plane $x y$ along the direction of $z$ axis and rise to three-dimensional cube $x y z$, so the calculation amount is $n_{z} \cdot S$.
Above all, the cell body segmentation method is used to conduct grid discretization of the workspace, and the calculation amount is $\left(n_{x}+n_{y}+n_{z}\right) \cdot S$, the calculation amount is significantly reduced compared with the traversal algorithm.

\subsection{Establishment of Robot Joint Coordinate System}

According to the configuration of the rehabilitation robot in the research group, the $\mathrm{DH}$ coordinate system of the robot is established.

As shown in Fig. 2, its coordinate parameters can be known in detail from Tab.1. Where $\theta_{i}$ represents the angle value of joint $i, d_{i}$ represents the length of links between adjacent joints, $a_{i}$ represents the offset of links between adjacent joints, and $\alpha_{i}$ represents the torsion angle between adjacent coordinate systems.

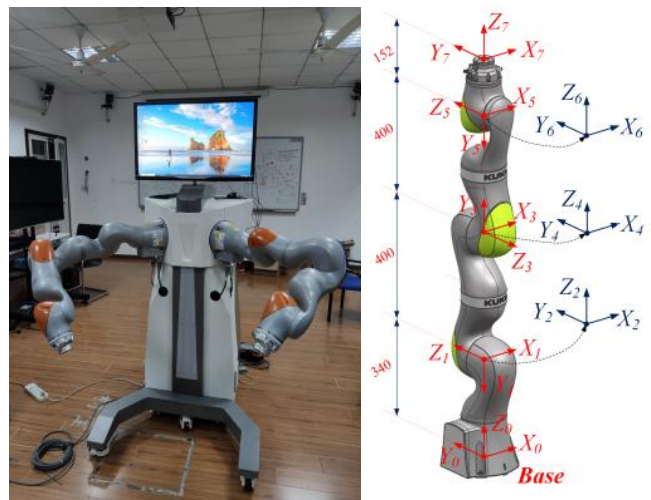

Fig. 2. DH coordinates of dual limb

Table 1. DH parameters of iiwa

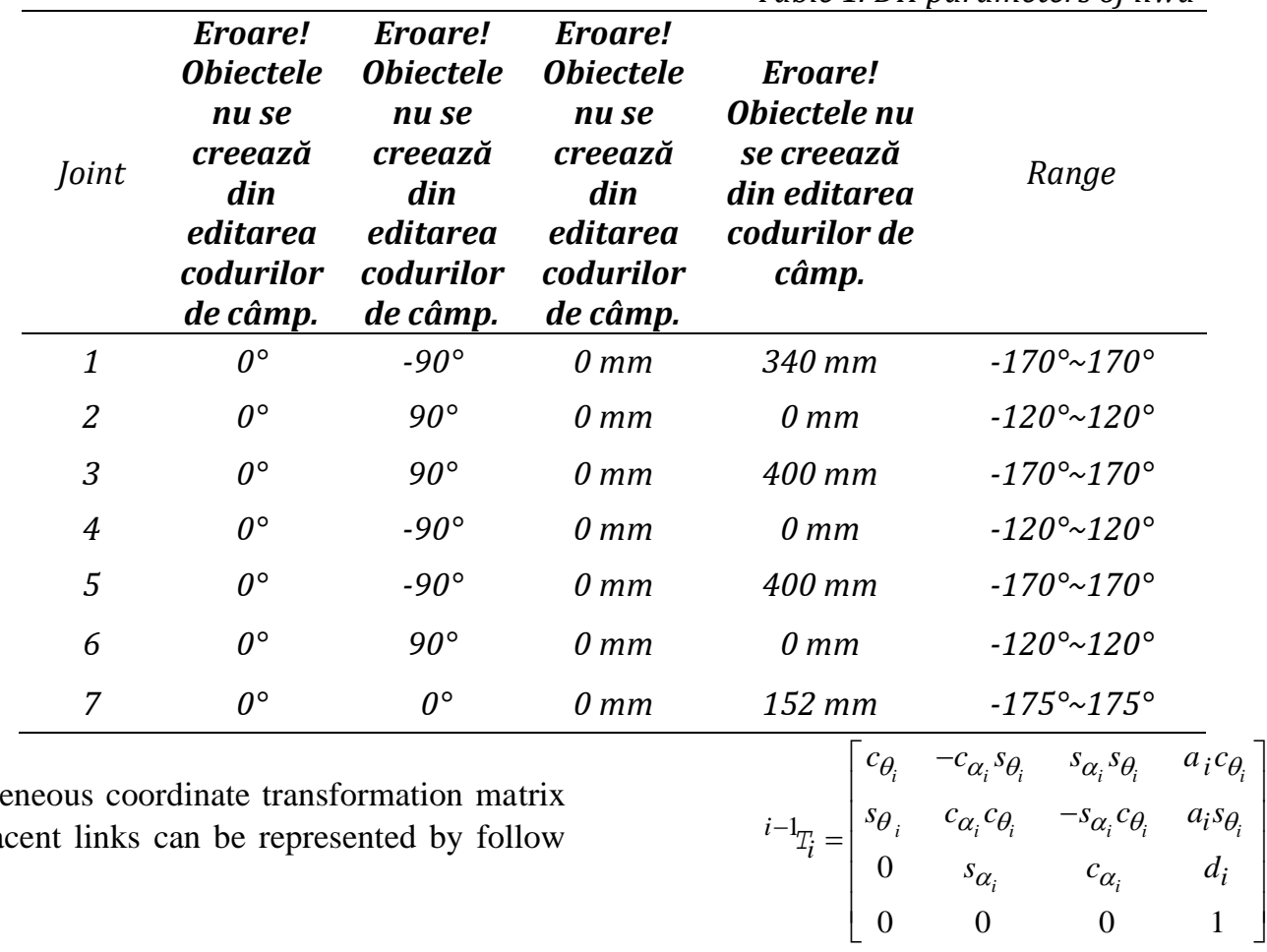
$T$ between adjacent links can be represented by follow equation. 
where $c_{\theta_{i}}$ represents $\cos \left(\theta_{i}\right), c_{\alpha_{i}}$ represents $\cos \left(\alpha_{i}\right)$, $s_{\theta_{i}}$ represents $\sin \left(\theta_{i}\right), s_{\alpha_{i}}$ represents $\sin \left(\alpha_{i}\right)$.

\subsection{Discrete Space of Rehabilitation Robot}

According to the rehabilitation robot configuration of the research group, the fixed reference system DH relation between the base of the robot and the base is established, which can be known in detail from Fig. 3, where $O_{W}-X_{W} Y_{W} Z_{W}$ represents the fixed reference system, $O_{O L}-X_{O L} Y_{O L} Z_{O L}$ represents the base frame of the robot left arm, $O_{O R}-X_{O R} Y_{O R} Z_{O R}$ represents the base frame of the robot right arm, and $\theta=45^{\circ}, L=334 \mathrm{~mm}$. According to the matrix Eq. (4) of the homogeneous coordinate transformation, the conversion relation between Eq. (5) and Eq. (6) is established, and then the fixed reference system relation between the robot endeffector and the frame is obtained. DH parameters in table 1 are combined to establish the forward kinematics equation of the robot.

$$
\begin{aligned}
& { }^{{ }^{w} T_{7 L}}={ }^{w} T_{0 L} 0 L_{T_{7 L}} \\
& { }^{{ }^{w} T_{7 R}}={ }^{w} T_{0 R} 0 L_{T_{7 R}}
\end{aligned}
$$

where ${ }^{w_{T}}{ }_{7 L}$ refers to the homogeneous rotation matrix between the end-effector frame of the left arm and the fixed reference system; ${ }^{w_{T}}{ }_{0 L}$ refers to the homogeneous rotation matrix between the base frame of the robot left arm and the fixed reference system; ${ }^{0 L_{T}}{ }_{7 L}$ refers to the homogeneous rotation matrix between end-effector frame of the left arm and the base frame of the robot left arm; ${ }^{w_{T_{7 R}}}$ refers to the homogeneous rotation matrix between the end-effector frame of the right arm and the fixed reference system; ${ }^{w_{T O R}}$ refers to the homogeneous rotation matrix between the base frame of the robot right arm and the world frame; and ${ }^{0 R_{T_{7 R}}}$ refers to the homogeneous rotation matrix between end-effector frame of the right arm and the base frame of the robot right arm.

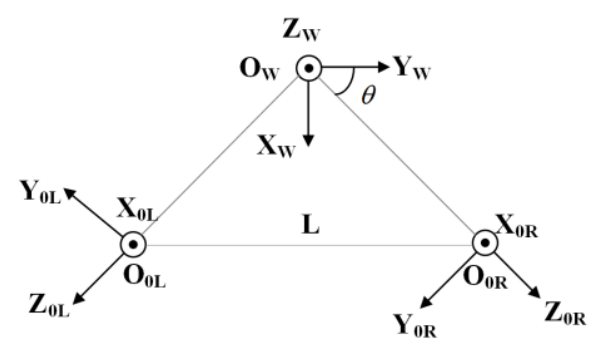

Fig. 3. Top view of the fixed reference system between the base of the robot and the base

Based on Robotics toolbox of the MATLAB, dual limb model is established according to $\mathrm{DH}$ parameters between connecting links. By Monte Carlo method and the robot kinematics, the dual limb rehabilitation robot workspace relative the fixed reference system can be obtained by the Eq. (1), Eq. (2), Eq. (5) and Eq. (6), and the discrete grid workspace can be calculated by using the cell segmentation method. The algorithm flow chart is as shown in Fig.4, by this way, we can get the discrete spatial grid graph of dual limb rehabilitation robot workspace, as shown in Fig.5.

Fig.5 (a) is the discrete space of dual limb stereogram, and Fig.5 (b) is the discrete space of partial sectional views, in which green areas are the left arm alone workspace, yellow areas are the right arm alone workspace, and blue areas are the cooperative space of dual limb.

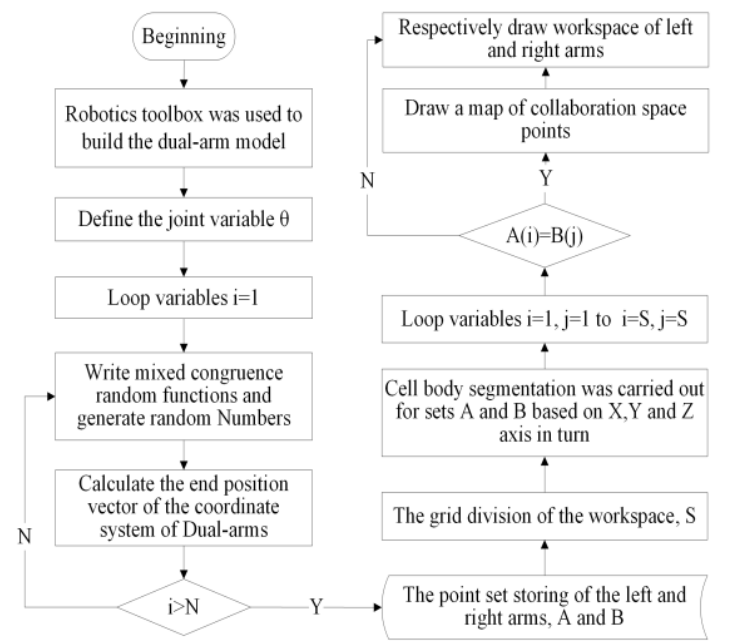

Fig.4. Block diagram of the workspace grid discrete

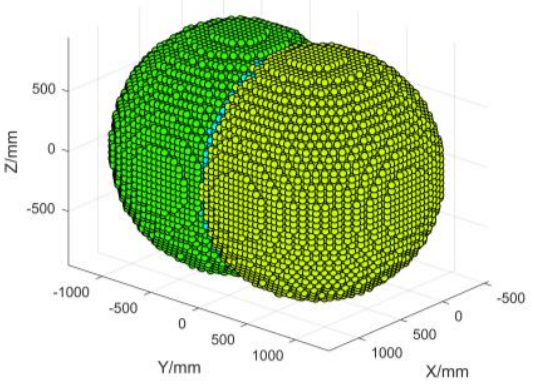

(a)

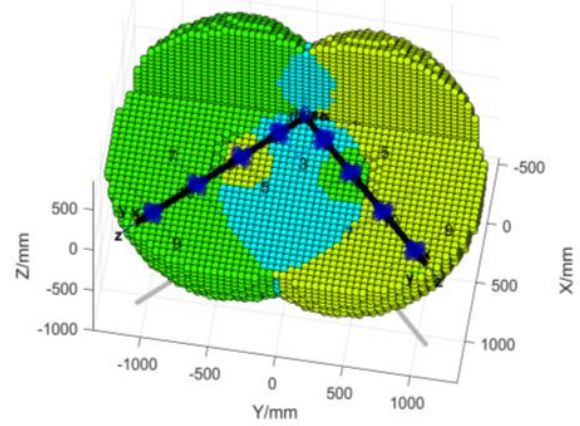

(b)

Fig.5. Discrete spatial grid diagram of rehabilitation robot 


\section{Kinematic Dexterity Analysis of the Robot}

\subsection{Analysis of Kinematic Dexterity}

The ratio between the angular velocity in joint space and the generalized velocity in Cartesian space is defined as Jacobian matrix [13], which can be represented by follow equation.

$$
\dot{x}=J(q) \dot{q}
$$

where $\dot{x}$ refers to Cartesian velocity vector; $J(q)$ refers to Jacobian matrix; $\dot{q}$ refers to the joint velocity vector.

In this study, since the research object only contains rotational variables, the Jacobian matrix of the revolute joint is calculated based on the speed of robot connecting link [14], and the formula is as follows

$$
J(q)=\left[\begin{array}{c}
z_{i-1} \times\left(p_{e}-p_{i-1}\right) \\
z_{i-1}
\end{array}\right]
$$

Then, the Jacobian matrix can be calculated

$$
J(q)=\left[\begin{array}{cccc}
z_{\mathrm{b}} \times\left(p_{7}-p_{\mathrm{b}}\right) & z_{1} \times\left(p_{7}-p_{1}\right) & \ldots & z_{6} \times\left(p_{7}-p_{6}\right) \\
z_{\mathrm{b}} & z_{1} & \ldots & z_{6}
\end{array}\right]
$$

where $p_{e}$ refers to the position coordinate of the robot end-effector; $p_{\mathrm{b}}=[0,0,1] ; p_{1-1}$ refers to the position coordinate of joint $i-1 ; z_{\mathrm{b}}=[0,0,1]$; and $z_{i-1}$ refers to the axis direction vector of the joint $i-1$.

Combining the forward kinematics equation of the robot and the parameters in Tab.1 and Eq. (9), the Jacobian matrix of the robot can be calculated.

The index of the robot kinematics dexterity is analysed by the manipulability, which can be calculated from Eq. (10)

$$
M(q)=\sqrt{\operatorname{det}\left(J(q) \cdot J^{T}(q)\right)}
$$

Then, the manipulability of each operating point can be obtained. Then, the manipulability of each grid point is averaged to represent the manipulability $\overline{M(\mathrm{q})}$ of the grid, it can be known from Eq. (11)

$$
\overline{M(\mathrm{q})}=\frac{\sum_{i}^{N} M_{i}(\mathrm{q})}{N}
$$

where $M_{i}(\mathrm{q})$ refers to the manipulability of the $i$ operating point in the grid; $N$ refers to the total number of operating points in the grid.

In order to visualize the kinematic dexterity of the robot in the workspace, it is necessary to normalize the manipulability of the discrete grid, as shown in Eq. (12)

$$
M_{i}^{\prime}=\frac{\bar{M}_{i}}{\max \left(\bar{M}_{1}, \bar{M}_{2}, \ldots, \bar{M}_{s}\right)}
$$

where $M_{i}^{\prime}$ refers to the manipulability of the grid at point $i$ after normalization; $\bar{M}_{i}$ refers to the manipulability of the $i$ grid; $s$ refers to the total number of grids.

\subsection{Dexterity Spatial Distribution of Robot Operating Points}

To explore rehabilitation robot's dexterity of each operation point in the workspace, according to each operation point calculated above in the working space of each grid, we calculate the manipulability of each grid based on the manipulability dexterity index through the Eq. (9), Eq. (10) and Eq. (11). Then, the operability of each grid is normalized by Eq. (12). Finally, the normalization processing manipulability is obtained in the workspace of discrete distribution, and the manipulability is evaluated the dexterity of rehabilitation robot in the workspace. The manipulability after normalized processing can reflect the relationship between the dexterity of each operation point in the workspace and the optimal dexterity point. The dexterity of the entire workspace of the rehabilitation robot is expressed through visual processing.

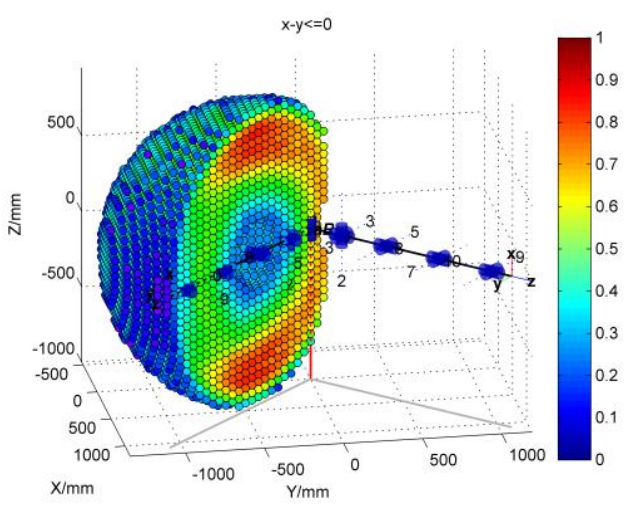

(a)

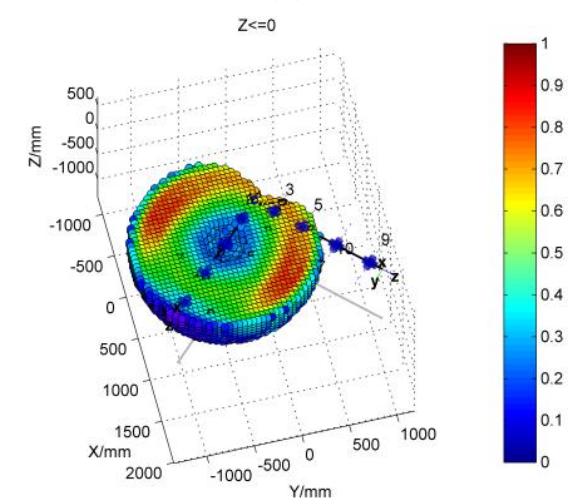


(b)

Fig. 6. A sectional view of the dexterity distribution in the left arm workspace

Combined with MATLAB visualization function, the dexterity distribution diagram of the rehabilitation robot in the grid discrete space is calculated by programming, as shown in Fig.6 and Fig.7. Fig.6 (a) is the section view of the left arm along plane $x-y=0$, and Fig. 6 (b) is the section view of the left arm along plane $\mathrm{Z}=0$.

Fig. 7 (a) shows the sections of the left arm and the right arm along plane $x-y=0$ and plane $x+y=0$. Fig.6 (b) shows the sections of the arms along plane $\mathrm{z}=0$. Each color in the figure represents the dexterity of the operating point and the range is $[0,1]$. The larger the coefficient, the more flexible it is. The robot in the red region has the best kinematic dexterity, while the robot in the blue region has the worst kinematic dexterity. It can be seen from Fig.6 and Fig. 7 that dexterity has a certain distribution rule in the workspace. In the center and edge area of the workspace, dexterity is poor, while in the middle zone area, dexterity is good, which will provide a reference for the selection and development of the movement trajectory of the rehabilitation robot.

In addition, due to the symmetry of the dual limb configuration of the rehabilitation robot, the kinematic dexterity discrete distribution of the dual limb should also be symmetrical. By combining Fig.7(a) and Fig.7(b), it is easy to conclude that the kinematic dexterity discrete distribution of the dual limb is symmetric with respect to the plane $x=0$, which can also verify the correctness of the hybrid method of Monte Carlo and cell segmentation based on manipulability.

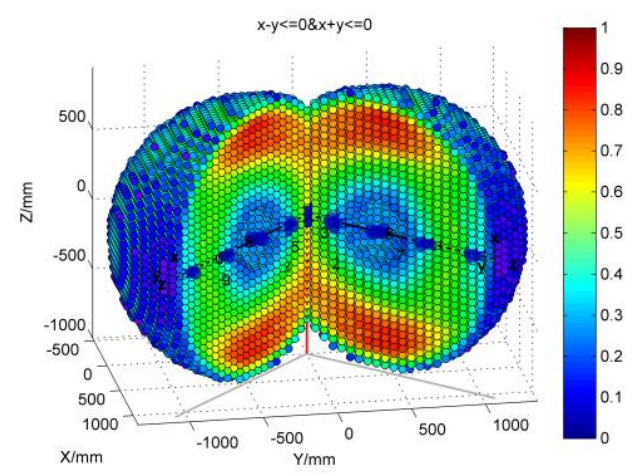

(a)

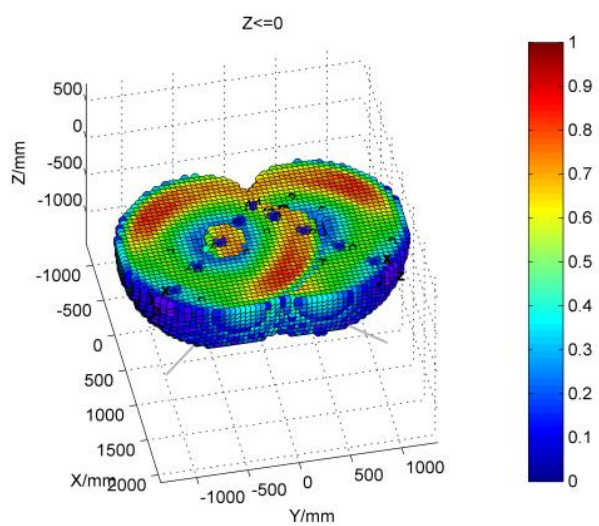

(b)

Fig.7. A sectional view of the distribution of dexterity in the dual limb workspace

\section{Conclusions}

In this study, the rehabilitation robot of the research group was studied. In order to avoid the inverse kinematics solution of the robot, the kinematic dexterity of the robot is analysed by Monte Carlo and spatial grid cell discrete mixing method based on the manipulability, and the flexible discrete distribution of the robot in the workspace is obtained. First, based on the Monte Carlo method, the robot is kinematically calculated to obtain the posture and corresponding operability of the robot end-effector. Then the grid of the robot's workspace is divided, and the operability of the points in each grid is averaged. Finally, the operability of each grid in the workspace is normalized, and the robot's movement dexterity is visualized based on MATLAB. Finally, the operability of each grid in the workspace is normalized, and the robot's movement dexterity is visualized based on MATLAB. Compared with the traditional traversal search, the calculation amount is also reduced. The visualization of the movement dexterity of the rehabilitation robot also provides a selection basis for the subsequent rehabilitation movement trajectory formulation.

\section{Acknowledgements}

This work was supported by the National Natural Science Foundation of China [Grant No.: 61973205] and National Key Research and Development Program of China [Grant No.: 2018YFC2001600].

\section{References}

[1] Battiston B, Titolo P, Ciclamini D, et al. Peripheral Nerve Defects: Overviews of Practice in Europe[J]. Hand Clinics, 2017, 33(3):545-550.

[2] French B, Thomas L H, Leathley M J, et al. Repetitive task training for improving 
functional ability after stroke[J]. Cochrane Database Syst Rev, 2007, 17(4): D6073.

[3] Azad M, Babič, Jan, Mistry M. Effects of the weighting matrix on dynamic manipulability of robots[J]. Autonomous Robots, 2019.

[4] Xie B. Directional Manipulability Constrained by the Condition Number[J]. Journal of Mechanical Engineering, 2010. 46(23): 8-15.

[5] Zargarbashi SHH, Khan W, Angeles J. The Jacobian condition number as a dexterity index in 6R machining robots[J]. Robotics and Computer-Integrated Manufacturing, 2012, 28(6): 694-699.

[6] Mansouri I, Ouali M. A new homogeneous manipulability measure of robot manipulators, based on power concept[J]. Mechatronics, 2009, 19(6):927-944.

[7] Chen C P, Wang P J, Wang H, et al. Developing industrial dual arm robot for flexible assembly through reachability map[J]. Technical Report, 2015.

[8] Wagner M, Peter Heß, Sebastian Reitelshöfer, et al. Reachability analysis for cooperative processing with industrial robots[C]// 2017 22nd IEEE International Conference on Emerging Technologies and Factory Automation. IEEE, 2017.
[9] Tian Yong, Wang Hongxing, Pan Xinan, et al. A Solving Method for the Workspace Dexterity of Collaborative Robot[J]. Robot, 2019, 041(003):298-306.

[10] Sun Q, Guo S, Zhang L. Kinematic dexterity analysis of Human-robot interaction of upper limb rehabilitation robot [J]. Technology and health care, 2020 (Preprint): 1-17.

[11] Liu Z, Liu H, Luo Z, et al. Improvement on Monte Carlo method for robot workspace determination[J]. Nongye Jixie Xuebao/transactions of the Chinese Society of Agricultural Machinery, 2013, 44(1):230-235.

[12] Li Xianhua, Tan shili, Huang Wuxin. A household service robot with two arms and multi-sensor in unstructured environments[C]. International Congress on Image and Signal Processing. Tianjin, China,2009: 4875-4879.

[13] Li Xianhua, Sun Qing, Zhang Leigang, et al. Dexterity analysis of coordination operation of dual-arm service robot[J]. Journal of Huazhong University of Science and Technology (Natural Science edition), 2019, 047(005):33-38.

[14] Bruno S, Lorenzo S, Luigi V, et al. Robotics: modeling, planning and control [M]. London: Springer-Verlag London Limited,2010: 105 113. 